ARTICLE

Received 4 Sep 2016 | Accepted 4 Apr 2017 | Published 26 May 2017 | Updated 13 Jul 2018 DOl: 10.1038/ncomms15587

\title{
Metabolic engineering of Saccharomyces cerevisiae for production of very long chain fatty acid-derived chemicals
}

Tao Yu ${ }^{1,2}$, Yongjin J. Zhou ${ }^{1,2, \dagger}$, Leonie Wenning ${ }^{1,2}$, Quanli Liu ${ }^{1,2}$, Anastasia Krivoruchko ${ }^{1,2,3}$, Verena Siewers ${ }^{1,2}$, Jens Nielsen ${ }^{1,2,4,5}$ \& Florian David ${ }^{1,2,3}$

Production of chemicals and biofuels through microbial fermentation is an economical and sustainable alternative for traditional chemical synthesis. Here we present the construction of a Saccharomyces cerevisiae platform strain for high-level production of very-long-chain fatty acid (VLCFA)-derived chemicals. Through rewiring the native fatty acid elongation system and implementing a heterologous Mycobacteria FAS I system, we establish an increased biosynthesis of VLCFAs in S. cerevisiae. VLCFAs can be selectively modified towards the fatty alcohol docosanol $\left(\mathrm{C}_{22} \mathrm{H}_{46} \mathrm{O}\right)$ by expressing a specific fatty acid reductase. Expression of this enzyme is shown to impair cell growth due to consumption of VLCFA-CoAs. We therefore implement a dynamic control strategy for separating cell growth from docosanol production. We successfully establish high-level and selective docosanol production of $83.5 \mathrm{mg} \mathrm{I}^{-1}$ in yeast. This approach will provide a universal strategy towards the production of similar high value chemicals in a more scalable, stable and sustainable manner.

\footnotetext{
${ }^{1}$ Department of Biology and Biological Engineering, Chalmers University of Technology, Kemivägen 10, Gothenburg SE-41296, Sweden. ${ }^{2}$ Novo Nordisk Foundation Center for Biosustainability, Chalmers University of Technology, Gothenburg SE-41296, Sweden. ${ }^{3}$ Biopetrolia AB, Systems and Synthetic Biology Group, Chalmers University of Technology, Kemivägen 10, Gothenburg SE-41296, Sweden. ${ }^{4}$ Novo Nordisk Foundation Center for Biosustainability, Technical University of Denmark, Kongens Lyngby DK-2800, Denmark. ${ }^{5}$ Science for Life Laboratory, Royal Institute of Technology, Stockholm SE-17121, Sweden. $\dagger$ Present address: Division of Biotechnology, Dalian Institute of Chemical Physics, Chinese Academy of Sciences, Dalian 116023, China. Correspondence and requests for materials should be addressed to F.D. (email: davidfl@chalmers.se).
} 
$\mathrm{V}$ ery-long-chain fatty acids (VLCFAs; 22-26 carbons) are essential biological components, which are incorporated in triacylglycerol molecules, sphingolipids, cuticle or waxes ${ }^{1}$. VLCFAs such as erucic acid, as well as VLCFA-derived products such as very-long-chain fatty alcohols (VLCFAlc) (for example, docosanol) and very-long-chain fatty waxes (for example, Jojoba oil) represent important classes of valuable chemicals, which are widely used as lubricants, detergents, polymers, photographic film-processing agents, coatings, cosmetics and pharmaceuticals ${ }^{2-5}$.

VLCFA chemicals are mainly extracted from natural sources or synthesized from petrochemical feedstocks. For natural sources, such as vegetable oils, the content of VLCFA derivatives is usually very low and only limited sources are available. When produced from petrochemical feedstocks, VLCFAs are synthesized chemically through oligomerization of ethylene followed by other modifications, which causes production cost increase with chain length ${ }^{6}$. Thus, a more scalable, stable and sustainable production route is needed. One promising solution is microbial production of VLCFAs and their derivatives from renewable feedstocks ${ }^{7}$. Here we engineered the FA metabolism for production of these VLCFA chemicals by the yeast Saccharomyces cerevisiae, a robust and industrially established microorganism for sustainable production of several products on the market ${ }^{8}$. As an initial target product we focused on docosanol, a saturated VLCFAlc with a chain length of 22 carbons, which is used as an emollient, emulsifier, thickener in cosmetics and nutritional supplements with a market volume of 40,000 ton per year. Furthermore, docosanol has been approved by the Food and Drug Administration as a pharmaceutical antiviral agent for reducing the duration of cold sores caused by the herpes simplex virus?.

Recently, multiple efforts have been initiated on engineering microbial FA synthesis for production of free FAs, alcohols, esters and alkanes ${ }^{10-15}$. Most of these studies have focused on products based on naturally abundant long-chain (LCFAs, 16-18 carbon) ${ }^{16}$, medium-chain (MCFAs, 6-14 carbon) or short-chain (SCFAs, $<6$ carbon $)^{17}$ FA-derived compounds. The particular chain length can be controlled at many steps during FA synthesis. In yeast, $\mathrm{C}_{16} / \mathrm{C}_{18}$ acyl-CoAs are generated by a type I FA synthase (FAS) system, a multimeric enzyme composed of Fas1 and Fas2 subunits in an $\alpha_{6} \beta_{6}$ complex $^{16}$. Further elongation to VLCFAs is carried out by membrane-embedded enzymes at the endoplasmic reticulum by a four-step process of condensation, reduction, dehydration and reduction ${ }^{18}$. One unit of malonyl-CoA is added after each cycle. In bacteria, FA synthesis is carried out by a type II FAS system, consisting of several separate enzymes. Mycobacteria harbour a special type I FAS I for the biosynthesis of VLCFAs, which are used as building blocks for the synthesis of mycolic acids, major constituents of their cell envelope with a chain length up to 90 carbons. The mycobacterial FAS I is a large multifunctional enzyme, which catalyses the de novo synthesis of FAs and shares homology with fungal $\mathrm{FAS}^{19}$. It produces both $\mathrm{C}_{16} / \mathrm{C}_{18} \mathrm{FAs}$ for the intrinsic cellular demand, as well as $\mathrm{C}_{22} / \mathrm{C}_{24} / \mathrm{C}_{26}$ FAs for the specific cell wall structures (Supplementary Table 1$)^{20}$.

Unlike LCFA-, MCFA- or SCFA-derived products, total biosynthesis of VLCFA-derived products encounters significant technical challenges: first, the chain length and amount of VLCFAs is controlled by strict cellular regulation mechanisms ${ }^{21}$; second, considering the longer chain length, VLCFAs biosynthesis needs much more reduction equivalents and ATP, which will cause a higher metabolic burden; third, the low concentration of cellular VLCFAs compared with $\mathrm{C}_{16} / \mathrm{C}_{18}$ FAs requires higher activity and specificity of the converting enzyme(s) to generate target products; fourth, the draining of VLCFAs towards the target compound biosynthesis can cause growth defects, as VLCFAs are necessary for essential cellular components such as sphingolipids and nuclear envelope structures $^{22}$. Thus, VLCFA-derived molecule production requires careful tuning and balancing of heterologous metabolic pathways and endogenous FA metabolism.

Here we successfully engineered yeast for the targeted production of VLCFAs and derived products thereof. As a first approach, we selectively modified the endogenous yeast FA elongation system to specifically control the chain length of VLCFAs. In a parallel approach, we demonstrated that the FAS I system from Mycobacterium vaccae can be functionally expressed in yeast generating a bimodal product distribution of $\mathrm{C}_{16-18}$ and $\mathrm{C}_{22-24}$ VLCFAs. Combining these two strategies, we could selectively produce VLCFAs ranging from $\mathrm{C}_{22}$ to $\mathrm{C}_{26}$ in chain length. Then, we further extended the metabolic pathways by expressing a $\mathrm{C}_{22}$-specific VLCFA reductase for the production of docosanol. To relieve the competition for VLCFAs between product formation and cell growth-associated processes, we implemented a dynamic control strategy for dividing the production phase and cell growth phase, which increased the docosanol production almost four-fold. Ultimately, by (re)constructing the whole pathway under this dynamic control, together with further enhancing the yeast elongation system, mycobacterial FAS I system and the precursor supply, we enabled a docosanol production of $83.5 \mathrm{mgl}^{-1}$, an almost 80 -fold improvement compared with the initial proof-of-concept strain. Our results show that the FA metabolism can be engineered for the production of VLCFA-derived chemicals in yeast. Furthermore, for the first time the mycobacterial FAS I system was successfully expressed in yeast for controlling the VLCFA chain length, representing a new and feasible strategy for targeted high-level production of VLCFAs and derived products (Fig. 1).

\section{Results}

VLCFA chain length control by FA elongation system. Normally, yeast generates very low amounts of VLCFAs $\left(\geq C_{20}\right)$. They are derived from the major FA components of $\mathrm{C}_{16} /{ }_{18} \mathrm{LCFAs}$ through several catalytic steps carried out by the intrinsic elongation system. $\beta$-Ketoacyl-CoA synthases, also known as elongases, catalyse the first and rate-limiting step of the elongation process by condensing acyl-CoA with malonyl-CoA building blocks ${ }^{23}$. These elongases determine the substrate specificity and final product profile of the whole elongation system $^{18}$. In S. cerevisiae, the elongases Elo1, Elo2 and Elo3 are required for VLCFA synthesis. These elongases have different substrate and product specificities: Elo1 has specificity for the elongation of $\mathrm{C}_{12-16}$ to $\mathrm{C}_{16-18} \mathrm{FAs}$, whereas Elo2 elongates $\mathrm{C}_{16-18}$ up to $C_{22}$ and Elo3 elongates $C_{18}$ up to $C_{26}$ (refs 18,24). We thus modulated these elongase genes for enhancing VLCFAs pools with chain length $\geq \mathrm{C}_{22}$.

We first engineered the VLCFAs biosynthesis in the LCFA-CoA overproducing strain JV03, in which the LCFACoA-consuming pathways including $\beta$-oxidation, triacylglycerol (TAG) and steryl ester synthesis were deleted (CEN.PK 113-5D

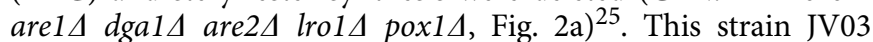
produced a small fraction of $\mathrm{C}_{26}$, whereas $\mathrm{C}_{22}$ and $\mathrm{C}_{24}$ VLCFAs were not detectable (Supplementary Fig. 1a). To drive the FA pool towards $\mathrm{C}_{22}$, we deleted the ELO3 gene to block $\mathrm{C}_{26}$ biosynthesis, which led to production of $\mathrm{C}_{22: 0}$ VLCFAs (strain TY001, Supplementary Figs $1 \mathrm{~b}$ and $2 \mathrm{~b}$ ). Subsequently, the ELO2 gene was overexpressed in JV03 elo3 $\Delta$ strain (TY002, Supplementary Table 5), which further increased the $C_{22}$ VLCFA level by almost 1.5 -fold (Fig. 2b). On the other hand, when overexpressing the ELO3 gene in the JV03 background strain TY003, the $\mathrm{C}_{26}$ VLCFA level increased almost twofold (Supplementary Fig. 1d). These 
Production of VLCFA-derived chemicals in yeast

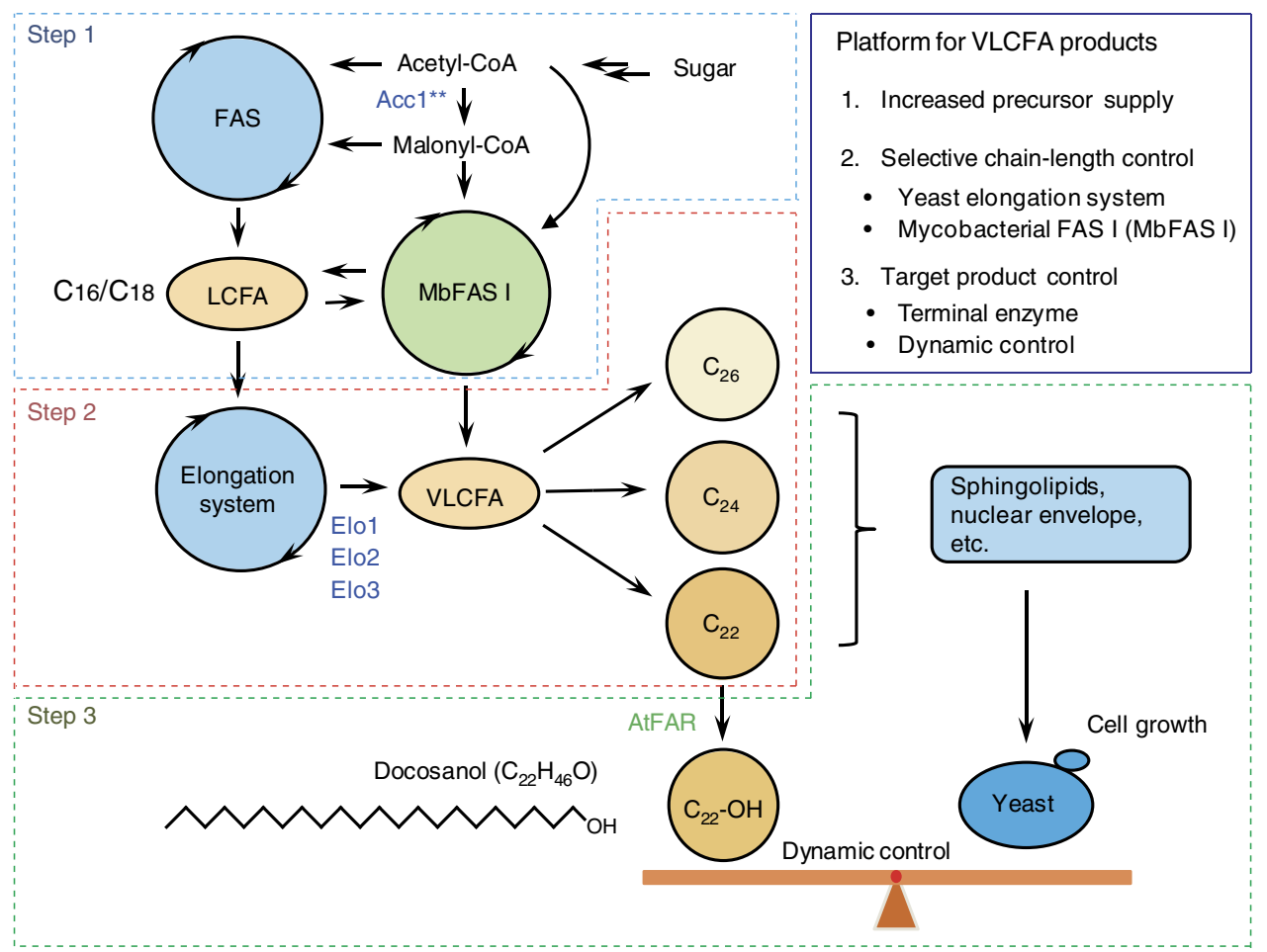

Figure 1 | Schematic overview describing platform technologies and toolboxes for targeted VLCFA and derived chemical production in yeast. LCFAs are specifically elongated to a VLCFA of choice via the intrinsic elongation system or Mycobacteria FAS I (MbFAS I) and further modified to the target product of interest. Product selectivity is determined by choice of enzymes and background strain.

results clearly showed that the chain length of VLCFAs could be selectively modulated by engineering the intrinsic yeast elongation system. Despite using an engineered strain, the overall production of C22 FAs was lower than $1 \mathrm{mgg}^{-1}$ dry cell weight (DCW).

VLCFAs chain length control by Mycobacterium FAS I system. To overcome the limitation of the intrinsic yeast elongation system, we focused on implementing the mycobacterial FAS system FAS I into yeast. In mycobacteria, this multimeric enzyme produces $\mathrm{C}_{16} / \mathrm{C}_{18} \mathrm{FAs}$ for the intrinsic cellular demand, as well as $\mathrm{C}_{22} / \mathrm{C}_{24} / \mathrm{C}_{26} \mathrm{FA}$ precursors for specific mycobacterial cell wall structures $^{20,26}$. We attempted to establish an additional source for VLCFAs production in yeast by expressing the $M$. vaccae FAS I gene (mvfas), which can specifically generate $\mathrm{C}_{22} / \mathrm{C}_{24}$, as well as $\mathrm{C}_{16} / \mathrm{C}_{18}$ FAs. The MvFAS was selected as it provides high levels of $\mathrm{C}_{22}$ VLCFAs as direct precursor for docosanol production. Compared with the multi-domain yeast Fas1/2 system, the MvFAS lacks the phosphopantetheinyl transferase domain and thus needs a separate acyl carrier protein synthase (Acps) to activate the ACP with phosphopantetheine. To guarantee a functional MvFAS, a cognate acps gene from M. vaccae was expressed in fusion with the carboxy-terminal mvfas gene via a $3^{*}$ GGGGS linker. This chimeric MvFAS-AcpS successfully restored the growth of a FA auxotrophic yeast strain PWY12

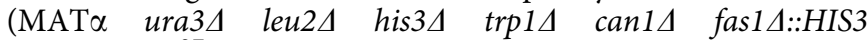
fas $2 \Delta:: L E U 2)^{27}$ on yeast extract peptone dextrose medium (YPD) or complete supplement mixture (CSM) synthetic media without FA addition (Supplementary Fig. 2). This result demonstrated that mvfas-acps was functionally expressed in $S$. cerevisiae. FA profile analysis showed that mvfas-acps expression in strain TY005 enabled a threefold increase in the
$\mathrm{C}_{26}$ VLCFA production level but only small amounts of $\mathrm{C}_{22}$ or $\mathrm{C}_{24}$ VLCFAs were produced (Supplementary Fig. 3). The high level of $\mathrm{C}_{26}$ VLCFA may be caused by the function of endogenous yeast elongation system through further elongation of the $\mathrm{C}_{22}$ and $\mathrm{C}_{24}$ FAs produced by the MvFAS. To avoid the interference, we expressed the $m v f a s$-acps gene in an elo2 $\Delta /$ elo3 $\Delta$ double knockout strain $^{28}$, which is growth deficient even with VLCFAs supplementation. Thus, an ELO3 expressing plasmid pELO3 was introduced to this strain for complementing the growth deficiency (strain TDY7005). To test the mycobacterial FAS system in this background strain, the plasmid p415GPD-MvFASAcpS was introduced into strain TDY7005. These transformants were screened for growth on 5-fluoroorotic acid (5-FOA) plates to obtain the strain TY004, which had lost the plasmid carrying the ELO3 gene (Fig. 2c). The results showed that mvfas-acps is able to replace the ELO3 gene in the elo24/elo3 4 background strain. Total FAs analysis of the strain TDY7005 showed that this strain also generated $\mathrm{C}_{26}$ VLCFAs due to the overexpression of ELO3 (Supplementary Fig. 4b), which is consistent with previous results (Supplementary Fig. 1c). However, the strain TY004, expressing a functional version of $m v f a s$-acps, produced high levels of $\mathrm{C}_{22}$ and $\mathrm{C}_{24}$ FAs as expected from the native activity of the enzyme (Fig. $2 c$ and Supplementary Fig. 4a).

These results show that the mycobacterial FAS I system is able to replace both the FA synthesis (FAS1/FAS2) and elongation (ELO2/ELO3) function in yeast. By eliminating intrinsic competing elongation reactions, we were able to selectively produce VLCFAs with chain length of $\mathrm{C}_{22}$ and $\mathrm{C}_{24}$. Expressing MvFAS led to almost fourfold higher production of $\mathrm{C}_{22}$ VLCFAs compared with engineering of the endogenous yeast elongation system (Fig. 2b,c). The increased production is likely to be the result of two factors: (1) MvFAS can generate $\mathrm{C}_{16}$ and $\mathrm{C}_{18}$ by itself and also can elongate the $\mathrm{C}_{16} / \mathrm{C}_{18}$ acyl-CoAs produced by the yeast 
a

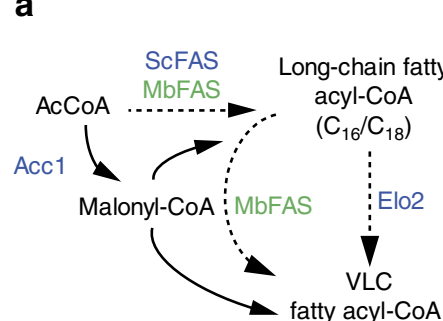

$$
\text { Aret,2 Steryl esters }
$$

Pox1

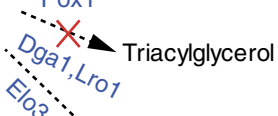

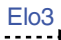

$\left(\mathrm{C}_{22}\right)$

VLC

fatty acyl-CoA

$\left(\mathrm{C}_{26}\right)$
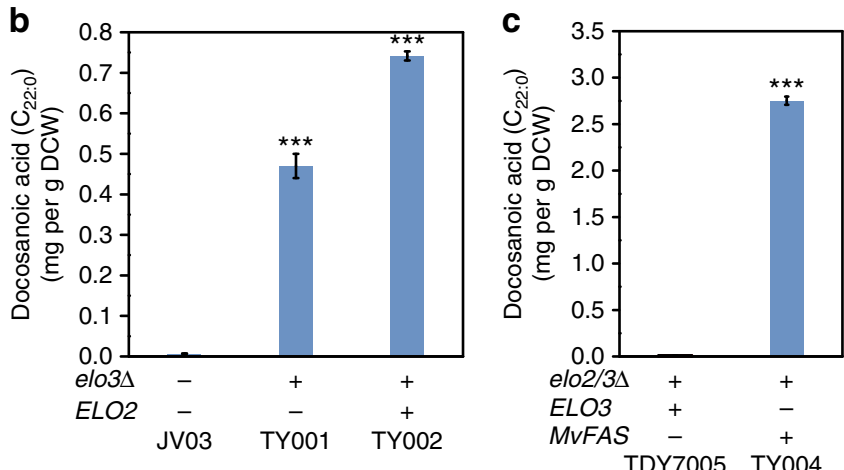

Figure 2 | Engineering production of $\mathbf{C}_{\mathbf{2 2}}$ VLCFAs by FA chain-length control in yeast. (a) Schematic overview of two strategies for chain length control towards VLCFAs. The dotted lines indicate multiple steps and solid lines a single step. Overexpressed genes are shown in blue (endogenous) or green (heterologous). Competitive pathways were eliminated by deleting corresponding genes (marked with X). Elo1, Elo2 and Elo3, yeast FA elongases 1, 2 and 3; MbFAS, FAS I system from Mycobacterium; ScFAS, S. cerevisiae FAS. (b) GC-MS analysis of docosanoic acid ( $C_{22: 0}$ FAME) generated by yeast elongation system in strains TY001 (JV03 elo3 $\Delta$ ) and TY002 (JV03 elo3 $\Delta$ pELO2). Statistical analysis was performed using a Student's $t$-test (one-tailed; ${ }^{\star} P<0.05$; ${ }^{\star \star} P<0.01$ and ${ }^{\star \star}{ }^{\star} P<0.001$; two-sample unequal variance). At least two independent measurements were performed for each experiment and the mean \pm s.d. of three biological replicates of a representative measurement is shown. (c) GC-MS analysis of docosanoic acid generated in strain TDY7005 (MATa lys2 ura3-52 trp1D leu2 $\Delta$ elo2 $\Delta::$ kanMX elo3 $\Delta:: T R P 1 / p E L O 3$ ) and strain TY004 (MATa lys2 ura3-52 trp1 1 leu2 $\Delta$ elo2 $\Delta:: k a n M X$ elo3 $\Delta:: T R P 1 / p G P D 415-M v F A S-A c p S)$. Statistical analysis was performed using a Student's $t$-test (one-tailed; ${ }^{\star} P<0.05,{ }^{\star \star} P<0.01$ and ${ }^{\star \star \star} P P<0.001$; two-sample unequal variance). At least two independent measurements were performed for each experiment and the mean \pm s.d. of three biological replicates of a representative measurement is shown. All cells were grown as described in experimental procedures.

FAS and (2) using the exogenous MvFAS circumvents the intrinsic regulation mechanisms. Thus, the mycobacterial FAS I system allows for efficient production of specific VLCFAs. In the future, other VLCFAs could be produced via selectively choosing the particular mycobacterial FAS system that corresponds to the VLCFA chain length desired, as summarized in Supplementary Table 1.

Selection of FA reductase for docosanol production. After establishing the VLCFAs biosynthesis platform, we aimed to engineer it towards docosanol production, a high-value VLCFAderived chemical (Fig. 3a). For this purpose, two main challenges have to be faced: first, the low level of VLCFAs asks for efficient 'terminal enzyme(s)' to convert VLCFAs towards relevant chemicals. Second, with much higher levels of cellular $\mathrm{C}_{16}$ and $\mathrm{C}_{18}$ fatty acyl-CoAs, the terminal enzyme(s) should have high catalytic specificity. For docosanol production, we tested five fatty a

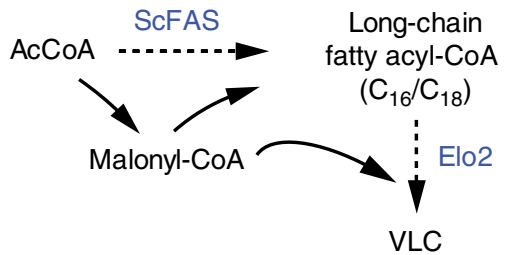

fatty acyl-CoA $\left(\mathrm{C}_{22}\right)$
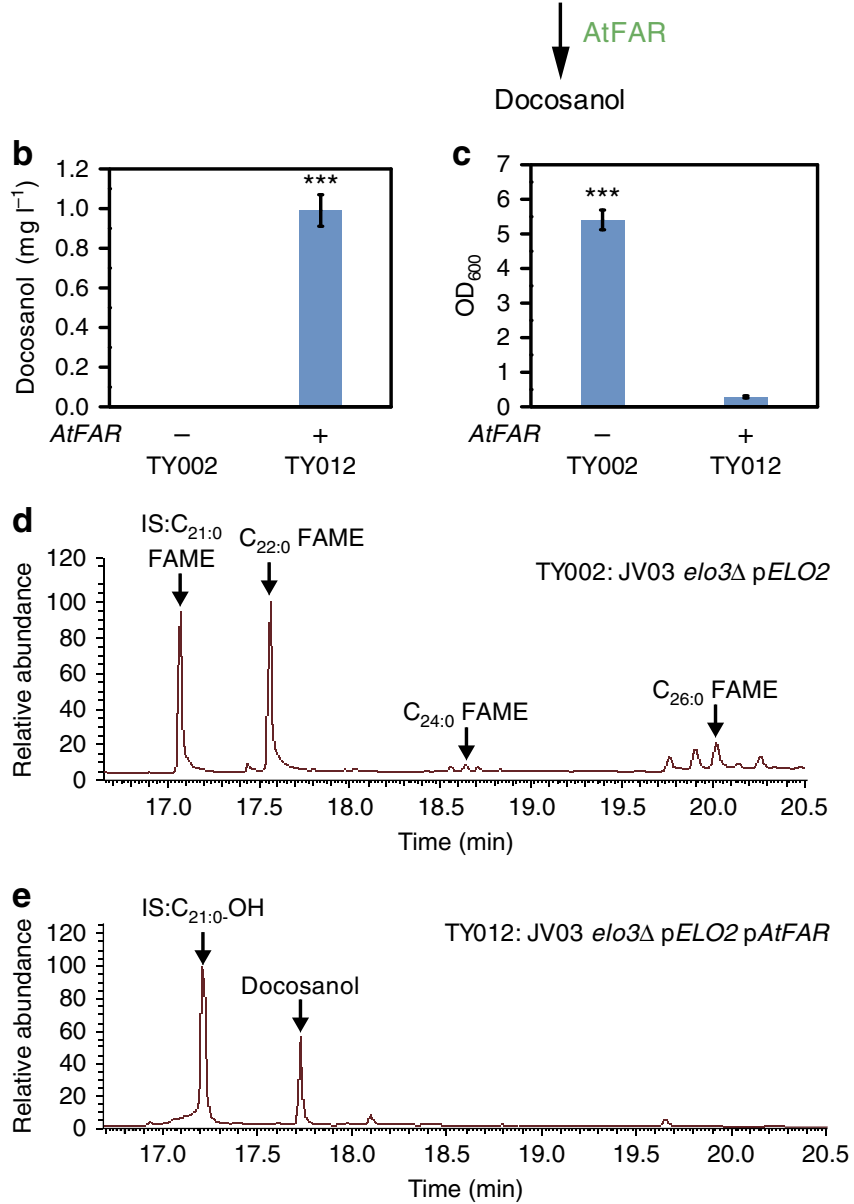

Figure 3 | Engineering production of docosanol $\left(\mathrm{C}_{22} \mathrm{H}_{46} \mathrm{O}\right)$ in yeast.

(a) Schematic biosynthetic pathway for docosanol. AtFAR, fatty acyl-CoA reductase from $A$. thaliana (AT5g22500). (b) GC-MS analysis of docosanol production in TY002 (JV03 elo3 pELO2) with or without expression of atfar. Statistical analysis was performed using a Student's t-test (one-tailed;

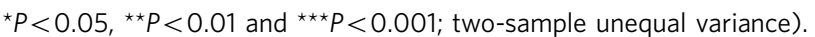
At least two independent measurements were performed for each experiment and the mean \pm s.d. of three biological replicates of a representative measurement is shown. (c) Final $\mathrm{OD}_{600}$ of strains TY002 and TY012, with or without expression of the fatty acyl-CoA reductase gene atfar. Statistical analysis was performed using a Student's $t$-test (one-tailed ${ }^{\star} P<0.05,{ }^{\star \star} P<0.01$ and ${ }^{\star \star \star} P<0.001$; two-sample unequal variance). At least two independent measurements were performed for each experiment and the mean \pm s.d. of three biological replicates of a representative measurement is shown. (d) VLCFA chain-length profiles of strain TY002 (JV03 elo3 $\Delta$ pELO2). IS (internal standard). (e) VLCFAlc profiles of docosanol producing strain TY012 (JV03 elo3s pVLCFAlc07). Typical GC-MS total ion chromatograms (TIC) of total FAs or fatty alcohols extracted from strain TYO02 (retention time from 16 to $21 \mathrm{~min}$ ) and strain TY012 (retention time from 16 to $21 \mathrm{~min}$ ). FAME and fatty alcohols were identified by retention time and comparison with the mass spectral library. All cells were grown as described under experimental procedures. 
acyl-CoA reductases that are capable of specifically converting VLCFA-CoAs towards VLCFAlcs (AmFAR from Apis mellifera ${ }^{29}$, AtFAR from Arabidopsis thaliana ${ }^{30}$, CfFAR from Calanus finmarchicus $^{29}$, ScFAR from Simmondsia chinensis ${ }^{30}$ and TaFAR from Triticum aestivum ${ }^{31}$, detailed description in Supplementary Table 8). We compared their activity in the strains with increased $\mathrm{C}_{22}$-CoA pools. TY001 (JV03 elo3 $\Delta$ ) and TY002 (JV03 elo3 $\Delta$ pELO2) were transformed with the respective plasmids resulting in strains TY006-TY015 (Supplementary Table 2). The fatty alcohol profile analysis revealed that most of the reductases tested did not show any detectable docosanol production, probably due to low conversion efficiency and specificity (Supplementary Table 8). Only atfar expression in strain TY002 led to the production of detectable amounts of $1.1 \mathrm{mgl}^{-1}$ docosanol (Fig. $3 \mathrm{~b}$ and Supplementary Table 2). Interestingly, no viable transformants were obtained when attempting to introduce atfar expressing plasmid to the strain TY001 (JV03 elo3A) (without ELO2 overexpression) (Supplementary Table 2). This might be attributed to the fact that AtFAR-deprived essential $\mathrm{C}_{22}$-CoA towards docosanol biosynthesis. ELO2 overexpression in strain TY012 (JV03 elo3A pVLCFAlc07) restored the cell growth possibly by providing higher levels of VLCFAs (Fig. 2b) for both docosanol biosynthesis and cell growth. Still, the biomass yield was much lower (final $\mathrm{OD}_{600}=0.3$ ) than for the control strain without expressing atfar (final $\mathrm{OD}_{600}=6.0$ ) (Fig. 3c). These results suggested that the docosanol biosynthesis deprived the cell of VLCFAs for cell growth. The metabolic flux therefore should be carefully balanced, to enable better cell proliferation and higher docosanol production. Furthermore, atfar expression in yeast resulted in specific production of docosanol (Fig. 3d,e), proving the high selectivity of this enzyme. This specificity was also shown previously in A. thaliana where a mutant of the atfar gene reduced the amount of $\mathrm{C}_{22: 0}-\mathrm{OH}$ in the suberin isolated from roots and sead coats by $30 \%$, whereas other fatty alcohols were not affected $^{32}$.

Dynamic control system to increase docosanol production. We successfully demonstrated the concept of docosanol production in yeast (Figs $3 \mathrm{~b}$ and $4 \mathrm{a}$ ). However, the titres remained very low due to the impaired cell growth caused by deprivation of essential precursors $\mathrm{C}_{22}$-CoA. Thus, we needed to fine tune the expression of these genes to balance cell growth and product formation. To reach this goal, we designed a dynamic control system where cell growth and docosanol production were separated into two different phases by using carbon source-dependent promoters (Fig. $4 \mathrm{~b}$ and Supplementary Fig. 8). To make the system as stringent and robust as possible, we tested the strategies in JV03 elo $3 \Delta$ strain. As a first attempt to increase cell growth with atfar expression, we expressed ELO3 under the control of HXT1 promoter, which is activated by high level of glucose and becomes repressed as the level of glucose decreases ${ }^{33}$. For atfar expression, we selected $H X T 7, A D H 2, I C L 1$ and GAL1 promoters ${ }^{33}$, which are repressed by high level of glucose and activated when the cells are in the ethanol phase or exposed to galactose as inducer, respectively (Fig. 4b). In addition, to evaluate constitutive expression of the atfar gene, we also tested the TDH3 and TEF1 promoters. The results of this screening are summarized in Supplementary Table 3 and highlighted in Fig. 4c (strains TY016-TY027). The strain TY018, having a deletion of ELO3 and atfar expressed under GAL1 promoter control, showed the highest docosanol production of $4.2 \mathrm{mgl}^{-1}$, which is fourfold higher compared with the previous proof-of-concept strain TY012 (Figs 3b and 4c). The biomass yield was almost $80 \%$ of the control strain TY001 (without atfar expression). Further expression of ELO3 under HXT1 promoter control completely restored the cell growth, but docosanol biosynthesis was totally abolished. In the strains carrying HXT1p-ELO3, tuning the atfar expression with promoters $H X T 7 \mathrm{p}$ or $A D H 2 \mathrm{p}$ resulted in docosanol production of 0.8 and $0.2 \mathrm{mgl}^{-1}$, respectively, which was much lower than the TY018 strain. These results indicated that ELO3 disruption is essential for supplying $\mathrm{C}_{22}-\mathrm{CoA}$ for docosanol production and a strict growth phase-dependent control of atfar is necessary for fine tuning cell growth and increasing final docosanol titres. In conclusion, the dynamic control of gene expression successfully solved the growth defect caused by precursor deprivation which laid the foundation for high-level production of docosanol.

Efficient pathway reconstruction for production of docosanol. Although FA metabolism was successfully engineered for docosanol production, the titre needs to be further improved. Owing to the non-functional FA storage and degradation, the growth rate and final cell biomass of the JV03 background strain are lower than that of wild-type strain ${ }^{25}$. We thus re-engineered FA elongation and reduction towards docosanol production in the more robust background strain CEN.PK113-5D. With the recently established CRISPR/Cas9 technology ${ }^{34,35}$ and modular pathway engineering ${ }^{36}$ for yeast genetic engineering, we could easily transfer our metabolic engineering strategy to different genetic background strains. Here we mainly focused on integrating the genes into chromosome instead of using episomal expression to get more stable genotypes ${ }^{37}$. We reconstructed the entire pathway for docosanol production including modifications regarding chain-length control, terminal FAR enzyme expression and further optimization using the dynamic control system (Fig. 5a). First, we deleted ELO3 gene and integrated the atfar gene under GAL1 promoter control at the site of ELO3 simultaneously; the resulting strain TY028 produced $0.65 \mathrm{mgl}^{-1}$ docosanol. Further overexpressing ELO2 gene under the control of GAL10 promoter (strain TY029) improved the docosanol production by 4 -fold $\left(2.4 \mathrm{mgl}^{-1}\right)$ compared with the previous strain TY028. To provide more acyl-CoA precursors for the endoplasmic reticulum-localized yeast elongation system, we overexpressed ELO1 gene under GAL7 promoter (strain TY030, Supplementary Fig. 9). However, this modification had only a marginal effect on docosanol production (Fig. 5b), suggesting that precursor supply for the elongation system in terms of acyl-CoAs seemed not to be the rate limiting step at this point. In parallel, we aimed to further increase FA production by integrating and overexpressing a constitutive active version of the $\mathrm{ACC}^{* *}$ enzyme $\left(A c c 1^{\text {S659A,S1157A })}\right.$ in strain TY028, thereby leading to an increased precursor supply of malonyl-CoA ${ }^{1,38}$. At the same time, we implemented the deletion of the GAL1 gene to avoid potential inducer consumption and ensure stable GAL promoter performance $^{39}$. The resulting strain TY033 could generate $1.4 \mathrm{mgl}^{-1}$ docosanol, more than a twofold increase compared with the previous strain TY028. If further combined with the overexpression of ELO2 and ELO1, the docosanol production was remarkably increased to $5.7 \mathrm{mgl}^{-1}$ (strain TY034) and $51 \mathrm{mgl}^{-1}$ (strain TY035), respectively (Fig. 5b). This highlights that upregulating the elongation system, especially ELO1, only becomes beneficial when sufficient malonyl-CoA precursors for FA production are provided. By implementing the MvFAS system, the production level of docosanol was further increased. TY036, which provided increased malonyl-CoA precursor supply through $A C C 1^{* *}$ overexpression, generated docosanol up to $40 \mathrm{mgl}^{-1}$. When combining both the yeast elongation system and the MvFAS strategy in strain TY037, the level of docosanol 
a
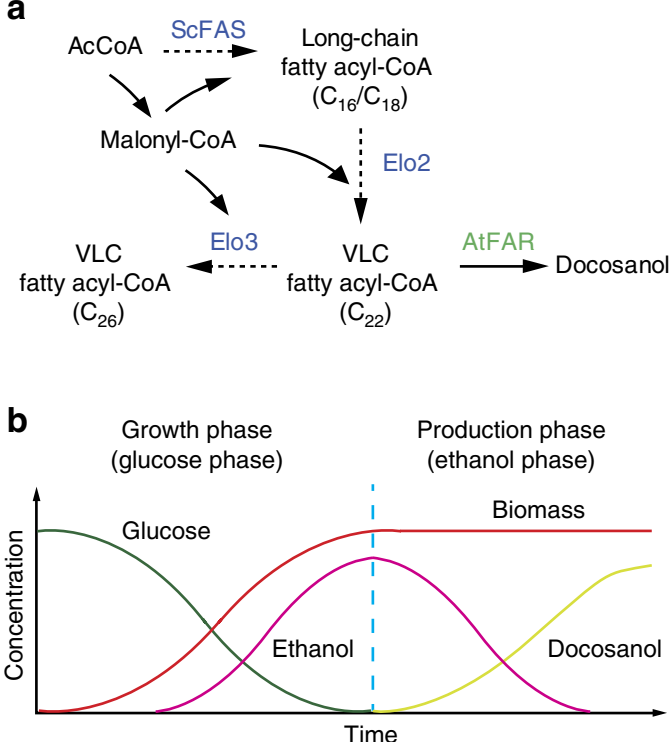

C
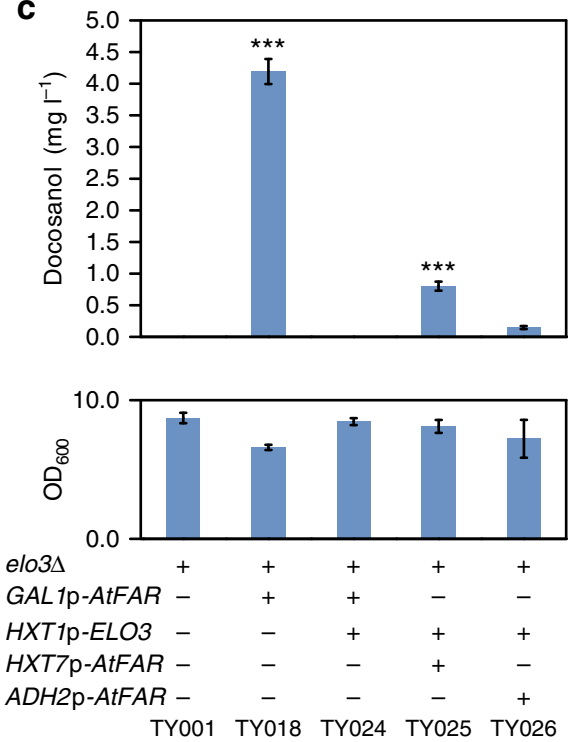

Figure 4 | Dynamic control of gene expression for increasing docosanol production. (a) Schematic pathway describing the reason for growth defect. (b) Schematic illustration describing the dynamic control system, where cell growth and docosanol production are separated into two different phases using carbon source dependent promoters. (c) Docosanol production and final culture $\mathrm{OD}_{600}$ in relevant strains. Statistical analysis was performed using a Student's $t$-test (one-tailed; ${ }^{\star} P<0.05,{ }^{\star \star} P<0.01$ and ${ }^{\star \star \star} P<0.001$; two-sample unequal variance). At least two independent measurements were performed for each experiment and the mean \pm s.d. of three biological replicates of a representative measurement is shown. All cells were grown and induced as described under experimental procedures.
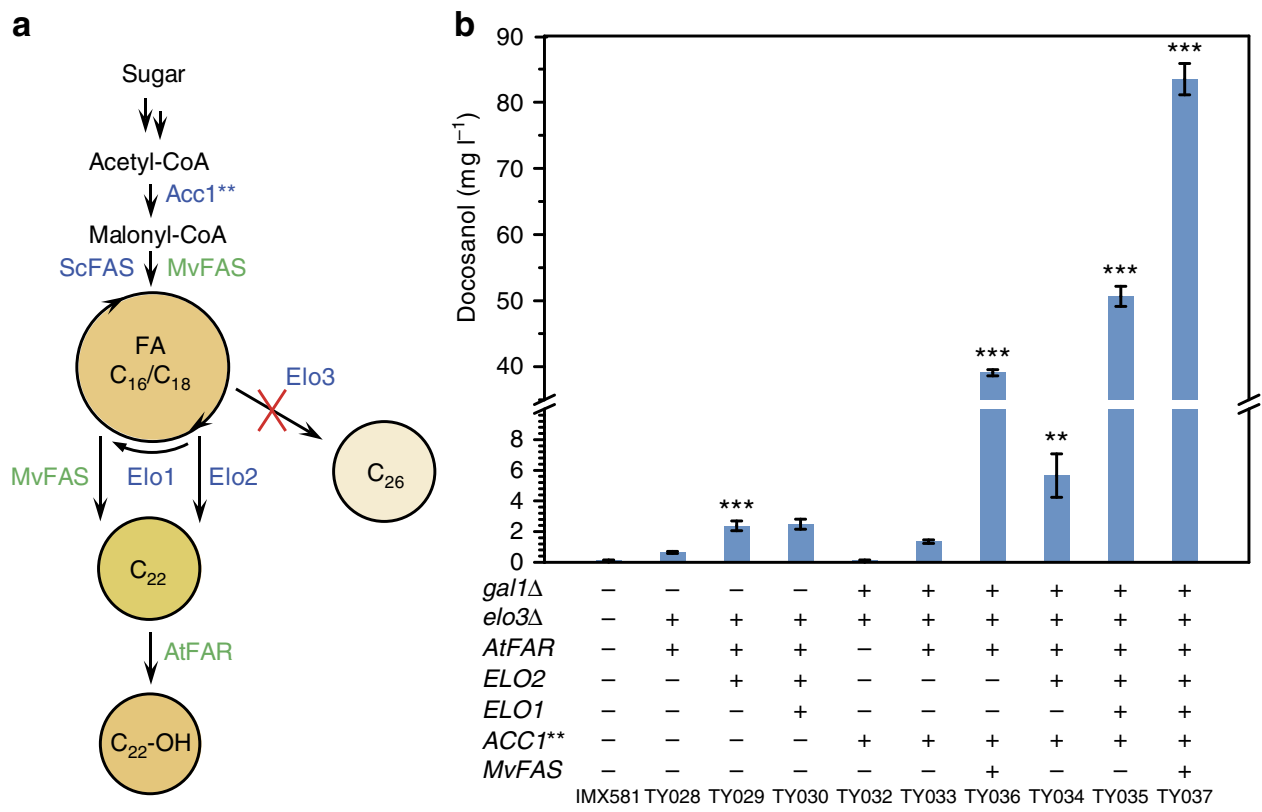

Figure 5 | High levels of docosanol production by reconstructed efficient pathway. (a) Schematic overview describing the applied strategy for increasing precursor supply and targeted docosanol production. (b) Docosanol production in CEN.PK113-5D background with stepwise applied modifications. All strains were grown in minimal medium with $0.5 \%$ galactose. All genes were integrated into the genome except mvfas, which was expressed from the plasmid pSPGM2-MvFAS-AcpS. Statistical analysis was performed using a Student's $t$-test (one-tailed; ${ }^{\star} P<0.05,{ }^{\star \star} P<0.01$ and ${ }^{\star \star \star} P<0.001$; two-sample unequal variance). At least two independent measurements were performed for each experiment and the mean \pm s.d. of three biological replicates of a representative measurement is shown.

was increased to $83.5 \mathrm{mgl}^{-1}$, an almost 80 -fold increase compared with the proof-of-concept strain TY012 (JV03 elo3 $\Delta$ pVLCFAlc07) (Figs 3b and 5b). Even after 80-fold improvement in production, docosanol is still the major fatty alcohol peak (Supplementary Fig. 5), demonstrating the high potential and selectivity of the implemented docosanol production pathway.

\section{Discussion}

VLCFAs and their derivatives are important precursors for valuable chemicals used in the food, drug, chemical and cosmetic industry. Here we established a yeast-based production platform for the production of high-value chemical docosanol. This was accomplished by overexpressing a highly active and specific FAR 
enzyme to generate VLCFAlcs and by controlling FA chain length towards VLCFAs.

During the last years there have been several reports on engineering FA metabolism for long-chain FA-derived chemical and biofuel production ${ }^{10,11,40-42}$ with the main focus on introducing heterologous pathways to transform cellular abundant FAs (mainly $\mathrm{C}_{16} /_{18}$ ). Here we succeeded to engineer FA metabolism towards VLCFA-derived chemical production. Unlike abundant $\mathrm{C}_{16} /_{18}$ FAs, the VLCFAs biosynthesis is strictly regulated, which makes it challenging to redirect the cellular metabolism towards the production of VLCFA-derived chemicals with specific chain length, such as docosanol. In our study, single modification of VLCFA biosynthesis using the mycobacterial MvFAS resulted in an accumulation of only $\mathrm{C}_{26} \mathrm{FA}$, but no $\mathrm{C}_{22}$ and $\mathrm{C}_{24}$ FAs (Supplementary Fig. 3). This suggests that the cellular elongation system is highly interconnected with the heterologous FAS. Thus, for the production of specific VLCFA chemicals, the intrinsic elongation system should be carefully tuned, depending on the target product of interest. Here we found that disruption of ELO3 and overexpression of ELO1 and ELO2 are the best combination for increased docosanol production. However, engineering the elongation system only produced small amounts of docosanol $\left(1.1 \mathrm{mgl}^{-1}\right.$, Fig. $\left.3 \mathrm{~b}\right)$. By additionally introducing the MvFAS system, as well as enhancing the precursor supply, we significantly improved docosanol production by 80 -fold to $83.5 \mathrm{mgl}^{-1}$ (Fig. 5b). A heterologous mycobacterial FAS I system was successfully constructed by fusing the phosphopantetheinyl transferase domain with the main FAS enzyme and was proven to be functional and efficient for VLCFA biosynthesis, and to enable high-level docosanol production in yeast (Fig. 5b).

Another challenge for producing VLCFA-derived chemicals is the need for a 'terminal enzyme' to selectively transform the precursor VLCFA-CoA into a relevant product, as VLCFA-CoA levels are much lower compared with $\mathrm{C}_{16} / \mathrm{C}_{18}$-CoAs. Thus, an efficient and specific FAR was selected, enabling the high level and targeted production of docosanol (Fig. 3e and Supplementary Fig. 5b). However, an efficient FAR might drain VLCFA-CoA precursors towards the end product, resulting in insufficient VLCFAs supply for cell growth. Correspondingly, we observed that constitutive expression of atfar decreased the biomass yield significantly (Fig. 3c). This is counterproductive as a certain amount of biomass is necessary to enable high-level production of the product of interest. The problem was solved by fine-tuning target gene expression using galactose-inducible promoters, thereby dynamically controlling the product pathway and separating the cell growth from the docosanol production phase. Targeted pathway overexpression and control via GAL promoters were previously used to optimize artemisinin production. The strategy was applied to avoid build-up of any potentially toxic intermediates in the mevalonate pathway, which may have resulted in strain instability ${ }^{39}$. Furthermore, in our study the strains benefited from this effect. When implementing the constitutively active version of the Accl enzyme, high levels of precursor are generated but at the same time this strain shows high instability ${ }^{10}$, possibly due to significant deprivation of the acetyl-CoA pool. To further improve docosanol production in the future, different strategies for increasing cytosolic acetyl-CoA supply could be evaluated ${ }^{43}$.

Recently, several studies showed the reconstruction of fatty acyl-CoA $\mathrm{C}^{16,44}$ or $\mathrm{FA}^{10}$ reduction pathways in S. cerevisiae, which enabled the production of about $100-300 \mathrm{mgl}^{-1}$ fatty alcohols with a chain-length mixture of $\mathrm{C}_{10}-\mathrm{C}_{18}$. Although facing additional challenges when producing VLCFAs and derived products, we reached a similar range in our studies with a final docosanol titre of $83.5 \mathrm{mgl}^{-1}$ with high purity.
These results clearly demonstrate that we were successful in establishing and combining three main platforms facilitating targeted VLCFAs and derived chemicals production in yeast (Fig. 1): (1) providing increased precursor supply for FA production, (2) tuning of yeast elongation system and choice of mycobacterial FAS I system for targeted chain length control of VLCFAs, and (3) choice of terminal enzyme and dynamic pathway control to generate the final product of interest. Our findings suggest that other chain length VLCFAs could be produced in a similar manner by tuning endogenous elongases, along with selection of the appropriate mycobacterial FAS I system. Depending on the background strains used and the terminal enzyme of choice, this technology would enable the production of various VLCFAs and derived products. These can be used for both bulk and specialty chemicals. In biobased production, the rate and yield on glucose are the main determinants for production costs, and hereby determining the cost competitiveness on the market. For low-priced bulk chemicals such as, for example, erucic acid, one has to operate near the theoretical yield, whereas for high-priced products, for example, waxes for cosmetics, lower yields are acceptable. At the same time, for products with higher chemical complexity more engineering has to be done to facilitate production. We show in this study that we were able to establish a production platform where we targeted several modifications, such as FA chain length and specific conversion to a C22 fatty alcohol, and even though we reach reasonable titres, further improvements are required to reach a commercial process.

However, our proof-of-principle demonstration that yeast can be used for production of long-chain fatty alcohols will facilitate the construction of microbial cell factories for the production of various new products in this area, in particular considering that industry favours the use of yeast as a robust and industrially established production host.

\section{Methods}

Strains and reagents. Primers, plasmids and S. cerevisiae strains used are listed in Supplementary Tables 4, 5 and 7. PrimeStar DNA polymerase was purchased from TaKaRa Bio. Taq DNA polymerase, restriction enzymes, DNA gel purification and plasmid extraction kits were purchased from Thermo Scientific. The Gibson Assembly Cloning Kit was purchased from NEB. Yeast plasmid Miniprep I kits were purchased from Zymo Research. All oligonucleotides (Supplementary Table 4) were synthesized at Sigma-Aldrich. All chemicals including analytical standards were purchased from Sigma-Aldrich, unless stated otherwise. All codon optimized heterologous genes were synthesized (Genscript) and listed in Supplementary Table 6.

Strain cultivation. Yeast strains for preparation of competent cells were cultivated in YPD consisting of $10 \mathrm{gl}^{-1}$ yeast extract (Merck Millipore), $20 \mathrm{gl}^{-1}$ peptone (Difco) and $20 \mathrm{gl}^{-1}$ glucose (Merck Millipore). Strains containing URA3 markerbased plasmids or cassettes were selected on synthetic complete media without uracil, which consisted of $6.7 \mathrm{gl}^{-1}$ yeast nitrogen base without amino acids (Formedium), $0.77 \mathrm{gl}^{-1} \mathrm{CSM}$ without uracil (Formedium), $20 \mathrm{gl}^{-1}$ glucose (Merck Millipore) and $20 \mathrm{gl}^{-1}$ agar (Merck Millipore). The URA3 maker was removed and selected against on synthetic complete media +5 -FOA plates, which contained $6.7 \mathrm{gl}^{-1}$ yeast nitrogen base, $0.77 \mathrm{gl}^{-1} \mathrm{CSM}$ and $0.8 \mathrm{gl}^{-1}$ 5-FOA. Strains containing the $k a n M X$ cassette were selected on YPD plates containing $200 \mathrm{mgl}^{-1} \mathrm{G} 418$ (Formedium). Shake flask batch fermentations for the production of fatty alcohols were carried out in minimal medium ${ }^{10,45}$ containing $30 \mathrm{gl}^{-1}$ glucose with or without $0.5 \%$ galactose supplemented with $60 \mathrm{mgl}^{-1}$ uracil if needed. Cultures were inoculated, from $24 \mathrm{~h}$ precultures, at initial $\mathrm{OD}_{600}$ of $0.05 \mathrm{in} 15 \mathrm{ml}$ minimal medium and cultivated at 200 r.p.m., $30^{\circ} \mathrm{C}$ for $72 \mathrm{~h}$. For $\Delta$ fas $1 / \Delta$ fas 2 strain, $\mathrm{C}_{16}(5 \mathrm{mM}$ palmitic acid $)$ and $\mathrm{C}_{18}(5 \mathrm{mM}$ stearic acid $)$ free FAs were dissolved in ethanol/Tween $20(1: 1)$ to get $100 \mathrm{ml} 100 \times$ FA stock medium, then sterilized by membrane filtration were and added as described before ${ }^{27}$.

Synthetic genes. S. cerevisiae codon-optimized synthetic genes of cffar (GenBank: AEO89345.1), atfar (NCBI reference sequence: NP_197642.1), amfar (NCBI accession number ADJ56408), scfar (NCBI accession number AF149917), tafar (TAA1a; NCBI accession number CAD30692), acps (NCBI reference sequence: 
WP_040539704.1) and mvfas (NCBI reference sequence: WP_003928293.1) were synthesized by GenScript, Inc. (Supplementary Table 6).

Construction of elo3 $\Delta:$ : $\boldsymbol{k a n M X}$. Oligonucleotide primers are listed in Supplementary Table 4. To delete ELO3, the Dell (LoxP-first half kanMX gene) fragment was amplified by primer pair pTY001/pTY002 using the plasmid pJET1.2-Del1 as template. The $502 \mathrm{bp}$ upstream of the ELO3 gene was amplified from CEN.PK113-11C genomic DNA with primer pair pTY005/pTY006. The upstream ELO3 region was fused by fusion PCR with the Dell fragment to obtain the fragment Upstream ELO3-LoxP-first half kanMX. The Del2 fragment (second half of kanMX gene ( + overlap)-GAL-promoter-Cre.recombinase-LoxP) was amplified by primer pair pTY003/pTY004 using the plasmid pJET1.2-Del2 as a template. The $500 \mathrm{bp}$ downstream of ELO3 gene was amplified from CEN.PK113$11 \mathrm{C}$ genomic DNA with primer pair pTY007/pTY008. The downstream ELO3 region was fused with the Del2 fragment to obtain Downstream ELO3 second half of kanMX gene ( + overlap)-GAL-promoter-Cre.recombinase-LoxP. Both fusion constructs were then used for co-transformation of strain JV03 to obtain strain TY001. The transformants were selected on G418 plates. Clones were verified by colony PCR using primer pairs pTY009/pTY002 and pTY010/pTY003. The ELO2 gene was amplified from CEN.PK113-11C genomic DNA with primer pair pTY024/pTY025. The backbone vector pSPGM2 was digested by BamHI. The ELO2 gene was ligated into the BamHI site of pSPGM2 to generate pELO2 by Gibson cloning. pELO2 was used to transform strain TY001 to generate TY002.

Construction of expression plasmid pSPGM2-MvFAS-AcpS. The pSPGM2MvFAS-AcpS was assembled on a yeast plasmid backbone pSPGM2 by using a modular pathway engineering strategy as described before ${ }^{46}$. In detail, the vector pSPGM2 was amplified by primer pair pTY011/pTY012. The whole expression cassette MvFAS-(GGGGS) $)_{3}$-AcpS was assembled using four parts: MvFAS2, MvFAS3, MvFAS4 and acps. These four parts have homologous overhangs among each other. In addition, MvFAS2 shared homologous overhangs with the TEF1 promoter of pSPGM2. The acps part shared homologous overhangs with the CYC1 terminator of pSPGM2. The MvFAS2 was amplified by primer pair pTY013/ pTY014. The MvFAS3 was amplified by primer pair pTY015/pTY016. The MvFAS4 was amplified by primer pair pTY017/pTY018. The acps was amplified by primer pair pTY019/pTY020. The primer pTY019 has the $3^{*}$ GGGGS sequence. All five parts were transformed into TDY7005 $(\Delta f a s 1 / \Delta$ fas 2$)$ and transformants were selected on YPD plates without any FA. Clones were verified by colony PCR. Subsequently, 3-4 clones with correct module integration were cultivated overnight in YPD liquid medium (Supplementary Fig. 6).

Functional replacement of ELO2/3 gene by MvFAS. The p415GPD-MvFASAcpS plasmid was assembled with a yeast plasmid backbone p415GPD by using a modular pathway engineering strategy as described before. In detail, the vector p415GPD was amplified by primers pTY022/pTY080. The whole expression cassette MvFAS-(GGGGS) - $_{3}$ AcpS was assembled in three parts: GPD-MvFAS2, MvFAS3 and MvFAS4-AcpS-CYC1t. These three parts have homologous regions between each other. The GPD (TDH3) promoter was amplified by primer pair pTY021/pTY022. The MvFAS2 was amplified by primer pair pTY023/pTY014. Then, the GPD promoter and MvFAS2 were fused together by overlapping PCR to obtain fragment GPD-MvFAS2. MvFAS3 was amplified with primer pair pTY015/ pTY016. MvFAS4-AcpS-CYC1t was amplified by primer pair pTY017/pTY081 using pSPGM2-MvFAS-AcpS as template. Then, all the five parts were transformed into TDY7005 $(\Delta$ elo2/Aelo3/pELO3) and transformants were selected on SD-LEU plates. These transformants were screened for growth on 5-FOA plates. Surviving clones were verified by colony PCR. Randomly, ten colonies were identified that had lost the plasmid that carried the URA3 marker and the ELO3 gene.

Construction of FAR expression plasmids. Codon-optimized synthetic genes of cffar, atfar, amfar, scfar and tafar were amplified by primer pairs pTY026/pTY027, pTY028/pTY029, pTY034/pTY035, pTY030/pTY031 and pTY032/pTY033, respectively. These FAR fragments were each ligated into the SpeI/NotI site of pSPGM2 to generate pVLCFAlc03, pVLCFAlc02, pVLCFAlc01, pVLCFAlc04 and pVLCFAlc05 via Gibson cloning. These plasmids were employed to transform TY001 (JV03 $\Delta$ elo3) to generate the strains TY008, TY007, TY006, TY009 and TY010. The respective FAR fragments were also ligated into the SpeI/NotI site of pELO2 to generate pVLCFAlc08, pVLCFAlc07, pVLCFAlc06, pVLCFAlc09 and pVLCFAlc10 via Gibson cloning. These plasmids were used to transform TY001 (JV03 $\Delta$ elo3) to generate the strains TY013, TY012, TY011, TY014 and TY015 (Supplementary Fig. 7).

Construction of plasmids for expression of AtFAR. The promoters $A D H 2 \mathrm{p}$, ICL1p, GAL1p, TEF1p, TDH3p and HXT7p were amplified from CEN.PK113-11C genomic DNA with primer pairs pTY036/pTY038, pTY039/pTY041, pTY042/ pTY044, pTY045/pTY060, pTY049/pTY062 and pTY047/pTY064 separately. The atfar gene was amplified by the primer pair pTY051/pTY052. The fragment FBA1t was amplified from CEN.PK113-11C genomic DNA with the primer pair pTY053/pTY054. The plasmid p416GPD was digested by SacI and KpnI, and the promoter $A D H 2 \mathrm{p}$; the amplified atfar fragment and the $F B A 1$ terminator were ligated into the SacI/KpnI site of p416GPD to generate the plasmid pDynCon05 via Gibson assembly. The same strategy was used to construct plasmids pDynCon06, pDynCon03, pDynCon02, pDynCon01 and pDynCon04. These plasmids were used to transform TY001 (JV03 $\Delta$ elo3) to generate the strains TY020, TY021, TY018, TY017, TY016 and TY019. To construct another set of plasmids, the promoters ADH2p, ICL1p, GAL1p, TEF1p, TDH3p and HXT7p were amplified from CEN.PK113-11C genomic DNA with primer pairs pTY037/pTY038, pTY040/ pTY041, pTY043/pTY044, pTY046/pTY060, pTY050/pTY062 and pTY048/ pTY064. The ELO3 gene was amplified from CEN.PK113-11C genomic DNA with primer pair pTY055/pTY056. The HXT1p promoter was amplified from CEN.PK113-11C genomic DNA with primer pair pTY057/pTY058. In the following, the fragments ELO3, HXT1p, $A D H 2 \mathrm{p}$, atfar and FBA1t were ligated into the SacI /Hindlll site of the p416GPD vector to generate the plasmid pDynCon 11 The same strategy was used to construct the plasmids pDynCon12, pDynCon09, pDynCon08, pDynCon 07 and pDynCon 10 . These plasmids were used to transform TY001 (JV03 Delo3) to generate strains TY026, TY027, TY024, TY023, TY022 and TY025 (Supplementary Fig. 8).

Reconstruction of the docosanol pathway. To select for specific genomic RNAs targeting for GAL1 and ELO3, all potential gRNAs were compared with all potential off-targets in the entire CEN.PK113-7D genome using the Yeastriction webtool $^{34}$, which is freely available at http://yeastriction.tnw.tudelft.nl. For deletion of GAL1, the $2 \mu \mathrm{m}$ fragment of pROS13 was amplified by primer pair pTY067/ pTY068. The linearized backbone of pROS13 was amplified by pTY102. The $2 \mu \mathrm{m}$ fragment and the backbone of pROS13 and were fused using Gibson Assembly to generate the gRNA expression vector pROS13-gRNA:GAL1. To repair the doublestrand break of GAL1, primers comprised of $2 \times 60$ bp sequences homologous to up- and downstream regions of GAL1 were used. The correct colonies were confirmed by primer pair pTY042/pTY073. To repair the double-strand break of GAL1 and integrate the $A C C 1^{* *}\left(A c c 1^{\mathrm{S} 659 \mathrm{~A}, \mathrm{~S} 1157 \mathrm{~A}}\right) \mathrm{gene}^{38}, A C C 1^{* *}$ was amplified by primer pair pTY071/pTY072, which comprised 100 bp overhang sequences homologous to up- and downstream regions of GAL1. The gRNA expression vector and repair fragment or primers were used to transform the IMX581 strain to generate strain TY031. Correct strains were confirmed by primer pairs pTY042/ pTY075 and pTY073/pTY074. For deletion of ELO3, the $2 \mu \mathrm{m}$ fragment of pROS10 was amplified by primer pair pTY069/pTY070. The linearized backbone of pROS10 was amplified by primer pTY102. The $2 \mu \mathrm{m}$ fragment and the backbone of pROS13 were fused using Gibson Assembly to get the gRNA expression vector pROS10gRNA:ELO3. The double-strand break of ELO3 was repaired by primer pair pTY065/pTY066, which comprised 60 bp sequences homologous to both up- and downstream regions of ELO3. The gRNA expression vector and repair primers were employed to transform strain TY031 to obtain TY032. Correct colonies were confirmed by primer pair pTY076/pTY077. To repair the double-strand break of ELO3 and integrate the atfar gene under control of the GAL1 promoter, the upstream fragment of ELO3 was amplified from CEN.PK113-11C genomic DNA with primer pair pTY094/pTY095. The GAL1p promoter was amplified from CEN.PK113-11C genomic DNA with primer pair pTY096/pTY097. The atfar gene was amplified by primer pair pTY087/pTY086 and the FBA1t terminator was amplified from CEN.PK113-11C genomic DNA with primer pair pTY088/pTY089. In addition, the downstream region of ELO3 was amplified from CEN.PK113-11C genomic DNA with primer pair pTY091/pTY092. Then, the five fragments were fused together by overlapping fusion PCR to obtain integration cassette ELO3upGAL1p-AtFAR-FBAt-ELO3dw. The gRNA expression vector and repair fragment were used to transform TY031 or IMX581 to generate the strains TY033 and TY028, respectively. To repair the double-strand break of ELO3 and integrate the atfar gene and $E L O 2$ gene, the upstream fragment of ELO3 was amplified from CEN.PK113-11C genomic DNA with primer pair pTY078/pTY079. In addition, the CYC1 terminator was amplified from CEN.PK113-11C genomic DNA with primer pair pTY080/pTY081 and the ELO2 gene was amplified from CEN.PK113 $11 \mathrm{C}$ genomic DNA with primer pair pTY082/pTY083. The bidirectional promoter GAL1p/GAL10p was amplified from CEN.PK113-11C genomic DNA with primer pair pTY084/pTY085. Atfar was amplified by primer pair pTY086/pTY087 and the FBA1t terminator was amplified by primer pair pTY088/pTY089. The downstream region of ELO3 was amplified from CEN.PK113-11C genomic DNA with primer pair pTY091/pTY092. Then, these fragments were fused together by overlapping PCR to generate the two integration cassettes ELO3up-CYC1t-ELO2-GAL10GAL1p and GAL10-GAL1p-AtFAR-FBA1t-ELO3dw. The gRNA expression vector and repair fragment were used to transform TY031 or IMX581 to generate the strains TY034 and TY029, respectively. To repair the double-strand break of ELO3 and integrate the atfar gene and ELO2, the upstream fragment of ELO3 was amplified from CEN.PK113-11C genomic DNA with primer pair pTY073/pTY093. The TDH2 terminator was amplified from CEN.PK113-11C genomic DNA with primer pair pTY103/pTY104. The ELO1 gene was amplified from CEN.PK113-11C genomic DNA with primer pair pTY105/pTY106. The GAL7 promoter was amplified from CEN.PK113-11C genomic DNA with primer pair pTY100/pTY101. The CYC1 terminator was amplified from CEN.PK113-11C genomic DNA with primer pair pTY080/pTY081. The ELO2 gene was amplified from CEN.PK113-11C genomic DNA with primer pair pTY082/pTY083. The bidirectional promoter GAL1p/GAL1p was amplified from CEN.PK113-11C genomic DNA with primer 
pair pTY084/pTY085. The atfar was amplified by primer pair pTY086/pTY087. The FBA1t terminator was amplified by primer pair pTY088/pTY089. The downstream region of ELO3 was amplified from CEN.PK113-11C genomic DNA with primer pair pTY091/pTY092. Then, these fragments were fused together by overlapping PCR to obtain the two integration cassettes ELO3up-TDH2t-ELO1GAL7p-CYC1t-ELO2 and ELO2-GAL10p-GAL1p-AtFAR-FBAt-ELO3dw. The gRNA expression vector and repair fragments were used to transform TY031 or IMX581 to generate strains TY035 and TY030, respectively. The strains TY033 and TY035 were transformed with plasmid pSPGM2-MvFAS-AcpS to generate the strains TY036 and TY037 (Supplementary Figs 9 and 10).

Total FA identification and quantification using GC-MS. Briefly, cell pellets were collected from $20 \mathrm{ml}$ cell culture and then freeze dried for $48 \mathrm{~h}$. Ten milligrams or $40 \mathrm{OD}_{600}$ of cell culture was prepared in an extraction tube. As extraction solvent, a solution of $1 \mathrm{ml}$ hexane and $2 \mathrm{ml}$ of $14 \% \mathrm{BF}_{3}$ in $\mathrm{MeOH}$ was used, which contained heneicosylic acid (C21:0, FA) as an internal standard. Subsequently, samples were flushed with $\mathrm{N}_{2}$ gas $(\sim 30 \mathrm{~s})$ to remove air and the lid was closed tightly. The transesterification was performed using a microwave treatment by programming the heating programme to increase from room temperature to $120^{\circ} \mathrm{C}$ (within $6 \mathrm{~min}$ ) and then keep the temperature at $120^{\circ} \mathrm{C}$ for $5 \mathrm{~min}$. Two millilitres of Milli-Q (MQ) water were added after the samples had been cooled down to room temperature. Samples were vortexed $(20 \mathrm{~s})$ and centrifuged at $2,000 \mathrm{~g}$ for $5 \mathrm{~min}$. The upper phase (hexane phase that contained the fatty acid methyl esters (FAMEs)) was transferred into a GC glass vials. The samples were analysed by gas chromatography (GC; Focus GC, Thermo Fisher Scientific, USA) equipped with a Zebron ZB-5MS GUARDIAN capillary column $(30 \mathrm{~m} \times 0.25 \mathrm{~mm} \times 0.25 \mu \mathrm{m}$, Phenomenex $)$ and a DSQII mass spectrometer (Thermo Fisher Scientific) ${ }^{47}$. The GC programme was as follows: initial temperature of $50^{\circ} \mathrm{C}$, hold for $5 \mathrm{~min}$; ramp to $140^{\circ} \mathrm{C}$ at a rate of $10^{\circ} \mathrm{C} \mathrm{min}{ }^{-1}$ and hold for $10 \mathrm{~min}$; ramp to $310^{\circ} \mathrm{C}$ at a rate of $15^{\circ} \mathrm{C} \mathrm{min}-1$ and hold for $7 \mathrm{~min}$. The GC programme for fatty alcohol quantification was as follows: initial temperature of $45^{\circ} \mathrm{C}$ hold for $2.5 \mathrm{~min}$; ramp to $220^{\circ} \mathrm{C}$ at a rate of $20^{\circ} \mathrm{C} \mathrm{min}^{-1}$ and hold for $2 \mathrm{~min}$; ramp to $300^{\circ} \mathrm{C}$ at a rate of $20^{\circ} \mathrm{C} \mathrm{min}^{-1}$ and hold for $5 \mathrm{~min}$. The temperature of inlet, mass transfer line and ion source were kept at $250{ }^{\circ} \mathrm{C}, 300^{\circ} \mathrm{C}$ and $230{ }^{\circ} \mathrm{C}$, respectively. The flow rate of the carrier gas (helium) was set at $1.0 \mathrm{ml} \mathrm{min}^{-1}$ and data were acquired at full scan mode $(50-650 \mathrm{~m} / \mathrm{z})$. Final quantification was performed with Xcalibur software.

Fatty alcohol identification and quantification. For fatty alcohol identification and quantification, cell pellets were collected from $5 \mathrm{ml}$ cell cultures and then freeze dried for $72 \mathrm{~h}$. As extraction solvent, a 2:1 chloroform:methanol solution was used, which contained heneicosanol as internal fatty alcohol standard. The extracted fraction was dried by rotary evaporation and dissolved in ethyl acetate ${ }^{10}$. Quantification of fatty alcohols was performed on the same gas chromatographymass spectrometry (GC-MS) system as used for FAME analysis or a GC-FID system (Thermo Fisher Scientific). The GC programme for fatty alcohol quantification was as follows: initial temperature of $45^{\circ} \mathrm{C}$ hold for $2.5 \mathrm{~min}$; then ramp to $220^{\circ} \mathrm{C}$ at a rate of $20^{\circ} \mathrm{C} \mathrm{min}^{-1}$ and hold for $2 \mathrm{~min}$; ramp to $300^{\circ} \mathrm{C}$ at a rate of $20^{\circ} \mathrm{C} \mathrm{min}^{-1}$ and hold for $5 \mathrm{~min}$. Especially for docosanol analysis, the following GC programme was used: initial temperature of $50^{\circ} \mathrm{C}$, hold for $5 \mathrm{~min}$ then ramp to $140{ }^{\circ} \mathrm{C}$ at a rate of $10^{\circ} \mathrm{C} \mathrm{min}-1$ and hold for $10 \mathrm{~min}$; ramp to $310^{\circ} \mathrm{C}$ at a rate of $15^{\circ} \mathrm{C} \mathrm{min}^{-1}$ and hold for $7 \mathrm{~min}$.

Data availability. The authors declare that all data supporting the findings of this study are available within the article and its Supplementary Information file or available from the corresponding author upon reasonable request.

\section{References}

1. Baud, S. et al. Multifunctional acetyl-CoA carboxylase 1 is essential for very long chain fatty acid elongation and embryo development in Arabidopsis. Plant J. 33, 75-86 (2003).

2. Taylor, D. C. et al. Brassica carinata-a new molecular farming platform for delivering bio-industrial oil feedstocks: case studies of genetic modifications to improve very long-chain fatty acid and oil content in seeds. Biofuel Bioprod. Bior. 4, 538-561 (2010).

3. Wisniak, J. Potential uses of jojoba oil and meal-a review. Ind. Crop Prod. 3, 43-68 (1994).

4. Miwa, T. K. Structural determination and uses of Jojoba oil. J. Am. Oil Chem. Soc. 61, 407-410 (1984).

5. Jannin, V. \& Cuppok, Y. Hot-melt coating with lipid excipients. Int. J. Pharmaceut. 457, 480-487 (2013).

6. Rupilius, W. \& Ahmad, S. Palm oil and palm kernel oil as raw materials for basic oleochemicals and biodiesel. Eur. J. Lipid Sci. Technol. 109, 433-439 (2007).

7. Feng, X. Y., Lian, J. Z. \& Zhao, H. M. Metabolic engineering of Saccharomyces cerevisiae to improve 1-hexadecanol production. Metab. Eng. 27, 10-19 (2015).

8. Jullesson, D., David, F., Pfleger, B. \& Nielsen, J. Impact of synthetic biology and metabolic engineering on industrial production of fine chemicals. Biotechnol. Adv. 33, 1395-1402 (2015).
9. Katz, D. H., Marcelletti, J. F., Khalil, M. H., Pope, L. E. \& Katz, L. R. Antiviral activity of 1-docosanol, an inhibitor of lipid-enveloped viruses including herpes simplex. Proc. Natl Acad. Sci. USA 88, 10825-10829 (1991).

10. Zhou, Y. J. J. et al. Production of fatty acid-derived oleochemicals and biofuels by synthetic yeast cell factories. Nat. Commun. 7, 11709 (2016).

11. Steen, E. J. et al. Microbial production of fatty-acid-derived fuels and chemicals from plant biomass. Nature 463, 559-U182 (2010).

12. Zhang, F. Z., Carothers, J. M. \& Keasling, J. D. Design of a dynamic sensor-regulator system for production of chemicals and fuels derived from fatty acids. Nat. Biotechnol. 30, 354-U166 (2012).

13. Schirmer, A., Rude, M. A., Li, X. Z., Popova, E. \& del Cardayre, S. B. Microbial biosynthesis of alkanes. Science 329, 559-562 (2010).

14. Zhou, Y. J. et al. Harnessing yeast peroxisomes for biosynthesis of fatty-acidderived biofuels and chemicals with relieved side-pathway competition. J. Am. Chem. Soc. 138, 15368-15377 (2016)

15. Zhou, Y. J., Buijs, N. A., Siewers, V. \& Nielsen, J. Fatty acid-derived biofuels and chemicals production in Saccharomyces cerevisiae. Front. Bioeng. Biotechnol. 2, 32 (2014).

16. Runguphan, W. \& Keasling, J. D. Metabolic engineering of Saccharomyces cerevisiae for production of fatty acid-derived biofuels and chemicals. Metab. Eng. 21, 103-113 (2014).

17. Torella, J. P. et al. Tailored fatty acid synthesis via dynamic control of fatty acid elongation. Proc. Natl Acad. Sci. USA 110, 11290-11295 (2013).

18. Tehlivets, O., Scheuringer, K. \& Kohlwein, S. D. Fatty acid synthesis and elongation in yeast. Biochim. Biophys. Acta 1771, 255-270 (2007).

19. Boehringer, D., Ban, N. \& Leibundgut, M. 7.5-angstrom cryo-em structure of the mycobacterial fatty acid synthase. J. Mol. Biol. 425, 841-849 (2013).

20. Kaneda, K., Imaizumi, S. \& Yano, I. Distribution of c-22-alpha-unit-containing, c-24-alpha-unit-containing and c-26-alpha-unit-containing mycolic acid homologs in Mycobacteria. Microbiol. Immunol. 39, 563-570 (1995).

21. Kihara, A. Very long-chain fatty acids: elongation, physiology and related disorders. J. Biochem. 152, 387-395 (2012).

22. Schneiter, R. et al. A yeast acetyl coenzyme a carboxylase mutant links verylong-chain fatty acid synthesis to the structure and function of the nuclear membrane-pore complex. Mol. Cell Biol. 16, 7161-7172 (1996).

23. Tvrdik, P. et al. Role of a new mammalian gene family in the biosynthesis of very long chain fatty acids and sphingolipids. J. Cell Biol. 149, 707-717 (2000)

24. Oh, C. S., Toke, D. A., Mandala, S. \& Martin, C. E. ELO2 and ELO3, homologues of the Saccharomyces cerevisiae ELO1 gene, function in fatty acid elongation and are required for sphingolipid formation. J. Biol. Chem. 272, 17376-17384 (1997).

25. Valle-Rodriguez, J. O., Shi, S. B., Siewers, V. \& Nielsen, J. Metabolic engineering of Saccharomyces cerevisiae for production of fatty acid ethyl esters, an advanced biofuel, by eliminating non-essential fatty acid utilization pathways. Appl. Energ. 115, 226-232 (2014).

26. Kikuchi, S., Rainwater, D. L. \& Kolattukudy, P. E. Purification and characterization of an unusually large fatty acid synthase from Mycobacterium tuberculosis var. bovis BCG. Arch. Biochem. Biophys. 295, 318-326 (1992).

27. Wenz, P., Schwank, S., Hoja, U. \& Schuller, H. J. A downstream regulatory element located within the coding sequence mediates autoregulated expression of the yeast fatty acid synthase gene FAS2 by the FAS1 gene product. Nucleic Acids Res. 29, 4625-4632 (2001).

28. Paul, S. et al. Members of the Arabidopsis FAE1-like3-ketoacyl-CoA synthase gene family substitute for the Elop proteins of Saccharomyces cerevisiae. J. Biol. Chem. 281, 9018-9029 (2006).

29. Teerawanichpan, P. \& Qiu, X. Molecular and functional analysis of three fatty acyl-CoA reductases with distinct substrate specificities in copepod Calanus finmarchicus. Mar. Biotechnol. 14, 227-236 (2012).

30. Rowland, O. \& Domergue, F. Plant fatty acyl reductases: enzymes generating fatty alcohols for protective layers with potential for industrial applications. Plant Sci. 193, 28-38 (2012).

31. Wang, Y. et al. Molecular characterization of TaFAR1 involved in primary alcohol biosynthesis of cuticular wax in hexaploid wheat. Plant Cell Physiol. 56, 1944-1961 (2015).

32. Domergue, F. et al. Three Arabidopsis fatty acyl-coenzyme A reductases, FAR1, FAR4, and FAR5, generate primary fatty alcohols associated with suberin deposition. Plant Physiol. 153, 1539-1554 (2010).

33. Keren, L. et al. Promoters maintain their relative activity levels under different growth conditions. Mol. Syst. Biol. 9, 701 (2013).

34. Mans, R. et al. CRISPR/Cas9: a molecular swiss army knife for simultaneous introduction of multiple genetic modifications in Saccharomyces cerevisiae. Yeast 32, S253-S253 (2015).

35. Jakounas, T. et al. Multiplex metabolic pathway engineering using CRISPR/Cas9 in Saccharomyces cerevisiae. Metab. Eng. 28, 213-222 (2015).

36. Zhou, Y. J. et al. Modular pathway engineering of diterpenoid synthases and the mevalonic acid pathway for miltiradiene production. J. Am. Chem. Soc. 134, 3234-3241 (2012). 
37. Jensen, N. B. et al. EasyClone: method for iterative chromosomal integration of multiple genes in Saccharomyces cerevisiae. Fems Yeast Res. 14, 238-248 (2014).

38. Shi, S. B., Chen, Y., Siewers, V. \& Nielsen, J. Improving production of malonyl coenzyme A-derived metabolites by abolishing Snf1-dependent regulation of Acc1. Mbio 5, e01130-14 (2014).

39. Westfall, P. J. et al. Production of amorphadiene in yeast, and its conversion to dihydroartemisinic acid, precursor to the antimalarial agent artemisinin. Proc. Natl Acad. Sci. USA 109, 111-118 (2012).

40. Chen, B. B., Lee, D. Y. \& Chang, M. W. Combinatorial metabolic engineering of Saccharomyces cerevisiae for terminal alkene production. Metab. Eng. 31, 53-61 (2015).

41. Buijs, N. A., Zhou, Y. J. J., Siewers, V. \& Nielsen, J. Long-chain alkane production by the yeast Saccharomyces cerevisiae. Biotechnol. Bioeng. 112, 1275-1279 (2015)

42. Liu, R. et al. Metabolic engineering of fatty acyl-ACP reductase-dependent pathway to improve fatty alcohol production in Escherichia coli. Metab. Eng. 22, 10-21 (2014).

43. van Rossum, H. M., Kozak, B. U., Pronk, J. T. \& van Maris, A. J. Engineering cytosolic acetyl-coenzyme A supply in Saccharomyces cerevisiae: pathway stoichiometry, free-energy conservation and redox-cofactor balancing. Metab. Eng. 36, 99-115 (2016).

44. Tang, X. L. \& Chen, W. N. Enhanced production of fatty alcohols by engineering the TAGs synthesis pathway in Saccharomyces cerevisiae. Biotechnol. Bioeng. 112, 386-392 (2015).

45. Verduyn, C., Postma, E., Scheffers, W. A. \& Van Dijken, J. P. Effect of benzoic acid on metabolic fluxes in yeasts: a continuous-culture study on the regulation of respiration and alcoholic fermentation. Yeast 8, 501-517 (1992).

46. Shao, Z. Y., Zhao, H. \& Zhao, H. M. DNA assembler, an in vivo genetic method for rapid construction of biochemical pathways. Nucleic Acids Res. 37, e16 (2009).

47. Khoomrung, S., Chumnanpuen, P., Jansa-ard, S., Nookaew, I. \& Nielsen, J. Fast and accurate preparation fatty acid methyl esters by microwave-assisted derivatization in the yeast Saccharomyces cerevisiae. Appl. Microbiol. Biotechnol. 94, 1637-1646 (2012).

\section{Acknowledgements}

This work was funded by the Novo Nordisk Foundation, Knut and Alice Wallenbergs foundation and the European Union's Horizon 2020 Framework Programme for Research and Innovation--Grant Agreement No. 720824, which are gratefully acknowledged. We thank Professor Teresa M. Dunn (Uniformed Services University, Bethesda, Maryland) for kindly sharing the elo2 4 elo3 $\Delta$ strain, Professor Hans-Joachim Schüller (University of Greifswald, Greifswald, Germany) for kindly sharing the fas 1$\triangle$ fas $\triangle 2$ strain, Alexandra Bergman for help with constructing the ELO3 deletion cassette,
Raphael Ferreira for help with constructing the CRISPR/Cas9 plasmid, Tyler Doughty for help with final polishing of the manuscript, and Sakda Khoomrung and Julia Karlsson for their help with GC analysis. We also appreciate the helpful discussion with Yun Chen Zhiwei Zhu, Mingtao Huang, Jiufu Qin, David Bergenholm, Boyang Ji, Yongjun Wei and Yi Liu.

\section{Author contributions}

T.Y. and F.D. conceived the study. T.Y. designed and performed all the experiments and analysed the data. F.D. and Y.J.Z. assisted with experimental design and data analysis. Q.L.L. assisted with experimental performance. L.W. assisted with constructing the ELO3 deletion strains. T.Y., F.D., Y.J.Z., L.W., Q.L.L., A.K., V.S. and J.N. wrote the manuscript.

\section{Additional information}

Supplementary Information accompanies this paper at http://www.nature.com/ naturecommunications

Competing interests: T.Y., F.D., Y.J.Z., L.W., A.K., V.S. and J.N. have filed a patent ('Fungal cells and methods for production of very long chain fatty acid derived products', number PCT/US/62/142,236) for protection of part of the work described herein. F.D., J.N., A.K. and V.S. are shareholders in Biopetrolia AB. All other authors declare no competing financial interests.

Reprints and permission information is available online at http://npg.nature.com/ reprintsandpermissions/

How to cite this article: Yu, T. et al. Metabolic engineering of Saccharomyces cerevisiae for production of very long chain fatty acid-derived chemicals. Nat. Commun. 8, 15587 doi: $10.1038 /$ ncomms15587 (2017)

Publisher's note: Springer Nature remains neutral with regard to jurisdictional claims in published maps and institutional affiliations.

(c) (i) This work is licensed under a Creative Commons Attribution 4.0 International License. The images or other third party material in this article are included in the article's Creative Commons license, unless indicated otherwise in the credit line; if the material is not included under the Creative Commons license, users will need to obtain permission from the license holder to reproduce the material. To view a copy of this license, visit http://creativecommons.org/licenses/by/4.0/

(C) The Author(s) 2017 


\section{Author Correction: Metabolic engineering of Saccharomyces cerevisiae for production of very long chain fatty acid-derived chemicals}

Tao Yu, Yongjin J. Zhou, Leonie Wenning, Quanli Liu, Anastasia Krivoruchko, Verena Siewers, Jens Nielsen \& Florian David

Nature Communications 8:15587 doi: 10.1038/ncomms15587 (2017); Published 26 May 2017, Updated 13 Jul 2018

In the originally published version of this Article, financial support was not fully acknowledged. The PDF and HTML versions of the Article have now been corrected to include support from the European Union's Horizon 2020 Framework Programme for Research and Innovation-Grant Agreement No. 720824.

(c) Open Access This article is licensed under a Creative Commons Attribution 4.0 International License, which permits use, sharing, adaptation, distribution and reproduction in any medium or format, as long as you give appropriate credit to the original author(s) and the source, provide a link to the Creative Commons license, and indicate if changes were made. The images or other third party material in this article are included in the article's Creative Commons license, unless indicated otherwise in a credit line to the material. If material is not included in the article's Creative Commons license and your intended use is not permitted by statutory regulation or exceeds the permitted use, you will need to obtain permission directly from the copyright holder. To view a copy of this license, visit http://creativecommons.org/licenses/by/4.0/

(C) The Author(s) 2018 